\title{
Method to Estimate the Position and Orientation of a Triaxial Accelerometer Mounted to an Industrial Manipulator
}

Patrik Axelsson and Mikael Norrlöf

Linköping University Post Print

N.B.: When citing this work, cite the original article.

Original Publication:

Patrik Axelsson and Mikael Norrlöf, Method to Estimate the Position and Orientation of a Triaxial Accelerometer Mounted to an Industrial Manipulator, 2012, 10th International IFAC Symposium on Robot Control, Dubrovnik, Croatia.

http://dx.doi.org/10.3182/20120905-3-HR-2030.00066

Postprint available at: Linköping University Electronic Press

http://urn.kb.se/resolve?urn=urn:nbn:se:liu:diva-81458 


\title{
Method to Estimate the Position and Orientation of a Triaxial Accelerometer Mounted to an Industrial Manipulator *
}

\author{
Patrik Axelsson, Mikael Norrlöf \\ Department of Electrical Engineering, Linköping University, \\ SE-58183 Linköping, Sweden (e-mail: \{axelsson, mino\}@isy.liu.se).
}

\begin{abstract}
A novel method to find the orientation and position of a triaxial accelerometer mounted on a six degrees-of-freedom industrial robot is proposed and evaluated on experimental data. The method consists of two consecutive steps, where the first is to estimate the orientation of the accelerometer from static experiments. In the second step the accelerometer position relative to the robot base is identified using accelerometer readings when the accelerometer moves in a circular path and where the accelerometer orientation is kept constant in a path fixed coordinate system. Once the accelerometer position and orientation are identified it is possible to use the accelerometer in robot model parameter identification and in advanced control solutions. Compared to previous methods, the accelerometer position estimation is completely new, whereas the orientation is found using an analytical solution to the optimisation problem. Previous methods use a parameterisation where the optimisation uses an iterative solver.
\end{abstract}

Keywords: Accelerometer, calibration, estimation, industrial robots

\section{INTRODUCTION}

The development in the area of industrial robotics is focused on, among other things, cost reduction leading to robots with less rigid mechanical structures. The main flexibilities in the mechanical system are considered to be introduced in the gearboxes. The current control strategy, using the motor angles for control of the robot, becomes insufficient with the new flexible structure. One of the possible solutions is to mount an accelerometer on the robot end-effector and estimate the joint angles on the arm side of the gearboxes (Axelsson et al., 2012; Axelsson, 2012). This gives a possibility to control the robot using an estimate of the complete system state. It is therefore essential to have good knowledge of the orientation and position of the accelerometer in order to get good estimates.

In this paper a novel method to estimate the position and orientation of a triaxial accelerometer mounted on an industrial robot is presented. The estimation method uses a two step procedure where the first step is to identify the orientation of the accelerometer using a number of static experiments. It is assumed that the accelerometer is mounted in such a way that it can be arbitrarily oriented using the six degrees-of-freedom (DOF) robot arm. The desired orientation of the accelerometer is hence known while the actual orientation is unknown. In Renk et al. (2005) and Won and Golnaraghi (2010) the accelerometer calibration is considered and internal parameters of the accelerometer, such as sensitivity and bias, but also alignment of each one of the three accelerometer measurement channels, are identified using an iterative search optimisation method. In this paper the unknown parameters

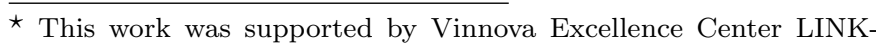
SIC. related to the accelerometer are found using an optimisation formulation where the solution can be expressed in closed form. By moving the robot such that centripetal acceleration can be measured, the method presented here also finds the position of the accelerometer. In Renk et al. (2005); Won and Golnaraghi (2010) it is also assumed that the accelerometer is moved, but it moves slowly and therefore only gravity affects the measurements.

The estimation problem is formulated in Section 2. In Section 3, the method to find the orientation of the accelerometer is described, and the method to estimate the mounting position is described in Section 4. The orientation and position estimation is evaluated on experimental data in Section 5 and Section 6 concludes the results.

\section{PROBLEM FORMULATION}

Assume that the accelerometer is mounted on the robot according to Figure 1(a) where the accelerometer is assumed to be rigidly attached to the robot tool. The problem addressed in this paper is to find:

(i) The internal sensor parameters and the orientation of the sensor.

(ii) The position of the accelerometer with respect to the robot tool coordinate system.

The manipulator has a spherical wrist, hence the orientation can be changed arbitrarily, only using the wrist joints. The estimation method finds the orientation and position of the triaxial accelerometer relative a given definition of the tool coordinate system. The orientation of the desired coordinate system can be seen in Figure 1(b). Let $\rho_{a}$ be an accelerometer measurement vector in the accelerometer coordinate system $O x_{a} y_{a} z_{a}$ and $\rho_{s}$ an acceleration vector 

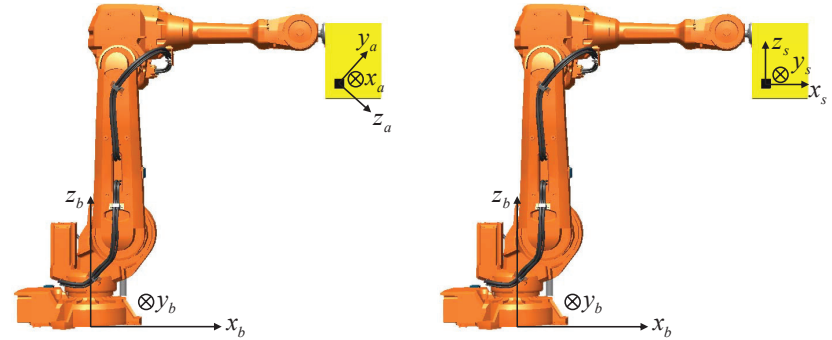

(a) The accelerometer and (b) The accelerometer and its actual coordinate system the desired coordinate system $O x_{a} y_{a} z_{a}$. $O x_{s} y_{s} z_{s}$.

Fig. 1. The accelerometer mounted on the robot. The yellow rectangle represents the tool or a weight and the black square on the yellow rectangle is the accelerometer. The base coordinate system $O x_{b} y_{b} z_{b}$ of the robot is also shown.

in the desired coordinate system $O x_{s} y_{s} z_{s}$, describing the acceleration in $\mathrm{m} / \mathrm{s}^{2}$. The relation between $\rho_{a}$ and $\rho_{s}$ is given by,

$$
\rho_{s}=\kappa R \rho_{a}+\rho_{0}
$$

where $R$ is the rotation matrix from $O x_{a} y_{a} z_{a}$ to $O x_{s} y_{s} z_{s}$, $\kappa$ is the accelerometer sensitivity and $\rho_{0}$ the bias. It is assumed that the same sensitivity value $\kappa$ can be used for all three sensors in the triaxial accelerometer. The sensitivity and bias is chosen such that the units in $O x_{s} y_{s} z_{s}$ are $\mathrm{m} / \mathrm{s}^{2}$. When the unknown parameters in (1) have been found the position of the accelerometer, expressed relative to the tool coordinate system, is identified. To solve for the unknown parameters, $\rho_{a}$ is measured while $\rho_{s}$ is computed from a model. When the robot is not moving, $\rho_{s}$ is simply the gravity vector, while in the dynamic case when the accelerometer is moved the acceleration will depend on the speed and orientation of the accelerometer.

\section{IDENTIFICATION OF ORIENTATION, SENSITIVITY AND BIAS}

To implement step (i) in Section 2, the gravity vector is measured in different orientations. The different orientations of the accelerometer are achieved by moving the robot tool to different orientations using the wrist joints of the robot, see Figure 2 for examples. To solve for the parameters $R, \kappa$ and $\rho_{0}$ in (1), first define the residual

$$
e_{k}=\rho_{s, k}-\kappa R \rho_{a, k}-\rho_{0},
$$

where $k$ indicates the sample number. Next, minimise the sum of the squared norm of the residuals,

$$
\begin{aligned}
\text { minimise } & \sum_{k=1}^{N}\left\|e_{k}\right\|^{2} \\
\text { subject to } & \operatorname{det}(R)=1 \\
& R^{T}=R^{-1}
\end{aligned}
$$

where the constrains guarantee that $R$ is an orthonormal matrix. By introducing the centroids for the measurements in $O x_{a} y_{a} z_{a}$ and $O x_{s} y_{s} z_{s}$

$$
\bar{\rho}_{s}=\frac{1}{N} \sum_{k=1}^{N} \rho_{s, k}, \quad \bar{\rho}_{a}=\frac{1}{N} \sum_{k=1}^{N} \rho_{a, k},
$$

and defining new coordinates,

$$
\rho_{s, i}^{\prime}=\rho_{s, i}-\bar{\rho}_{s}, \quad \rho_{a, i}^{\prime}=\rho_{a, i}-\bar{\rho}_{a}
$$

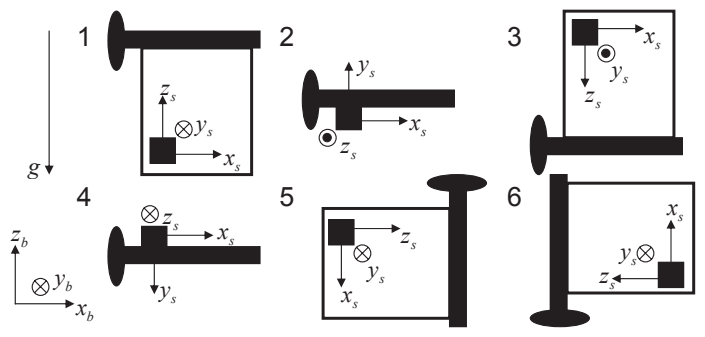

Fig. 2. Six different configurations of the robot tool used in Algorithm 1. The orientation of the desired coordinate system $O x_{s} y_{s} z_{s}$ is shown for each configuration. The base coordinate system $O x_{b} y_{b} z_{b}$ and the gravity vector are also shown.

the optimisation problem has the closed form solution (Horn et al., 1988),

$$
\begin{aligned}
\kappa & =\sqrt{\sum_{k=1}^{N}\left\|\rho_{s, k}^{\prime}\right\|^{2} / \sum_{k=1}^{N}\left\|\rho_{a, k}^{\prime}\right\|^{2},} \\
R & =M\left(M^{T} M\right)^{-1 / 2} \\
\rho_{0} & =\bar{\rho}_{s}-\kappa R \bar{\rho}_{a},
\end{aligned}
$$

where

$$
M=\sum_{k=1}^{N} \rho_{s, k}^{\prime}\left(\rho_{a, k}^{\prime}\right)^{T} .
$$

$N$ is the total number of measurements and it has to be assumed that $N \geq 3$. In addition a condition of sufficient excitement has to be fulfilled, such that $M^{T} M$ has full rank. As an alternative to the formulation above where the rotation is parameterised by the orthonormal matrix $R$ it is also possible to find a closed-form solution to (1) using unit quaternions, see e.g. Horn (1987). Considering the number of operations the matrix formulation is, however, computationally more efficient.

The orientation and the sensor parameters are found using static measurements, i.e., the robot is standing still in $N_{C}$ different tool orientation configurations. The gravity vector is measured by the accelerometer in each of the $N_{C}$ configurations, which gives $N_{M, j}, j=1, \ldots, N_{C}$ measurements for each configuration. Let

$$
\left\{\rho_{a}\right\}=\left\{\left\{\rho_{a, i}^{1}\right\}_{i=1}^{N_{M, 1}}, \ldots,\left\{\rho_{a, i}^{N_{C}}\right\}_{i=1}^{N_{M, N_{C}}}\right\}
$$

denote the set of all the $N=\sum_{j=1}^{N_{C}} N_{M, j}$ measurements in all $N_{C}$ configurations, and let

$$
\left\{\rho_{s}\right\}=\left\{\left\{\rho_{s}^{1}\right\}_{i=1}^{N_{M, 1}}, \ldots,\left\{\rho_{s}^{N_{C}}\right\}_{i=1}^{N_{M, N_{C}}}\right\}
$$

be the gravity vector from the model in the desired coordinate system $O x_{s} y_{s} z_{s}$ for each configuration, where $\rho_{s}^{j}, j=1, \ldots, N_{C}$ is a constant. The transformation parameters $R, \kappa$ and $\rho_{0}$ in (1) can be computed using the measured accelerations and the model values to solve the optimisation problem in (3) according to (4) to (7).

The $N_{C}$ different configurations can be chosen arbitrary as long as the matrix $M^{T} M$ has full rank ${ }^{1}$. Here six different configurations according to Figure 2 are suggested, which give

1 The matrix $M^{T} M$ has always full rank if none of the two sets $\left\{\rho_{a}\right\}$ and $\left\{\rho_{s}\right\}$ are coplanar. 


$$
\begin{aligned}
& \rho_{s}^{1}=\left(\begin{array}{lll}
0 & 0 & g
\end{array}\right)^{T}, \rho_{s}^{2}=\left(\begin{array}{lll}
0 & g & 0
\end{array}\right)^{T}, \\
& \rho_{s}^{3}=\left(\begin{array}{lll}
0 & 0 & -g
\end{array}\right)^{T}, \rho_{s}^{4}=\left(\begin{array}{lll}
0 & -g & 0
\end{array}\right)^{T}, \\
& \rho_{s}^{5}=\left(\begin{array}{lll}
-g & 0 & 0
\end{array}\right)^{T}, \rho_{s}^{6}=\left(\begin{array}{lll}
g & 0 & 0
\end{array}\right)^{T},
\end{aligned}
$$

where $g=9.81 \mathrm{~m} / \mathrm{s}^{2}$. Note that the vectors in (10) are directed towards the gravity vector in Figure 2. The explanation for this is that an accelerometer measures the normal force which is opposite the gravity vector.

The six configurations in Figure 2 are straightforward to obtain for a spherical wrist, six degree of freedom industrial manipulator (Sciavicco and Siciliano, 2000). The procedure to estimate the triaxial accelerometer sensor parameters is summarised in Algorithm 1.

Algorithm 1. Estimation of the sensor parameters

a) Measure the acceleration for the different configurations in Figure 2 to obtain $\left\{\rho_{a}\right\}$ according to (8).

b) Construct $\left\{\rho_{s}\right\}$ in (9) from (10).

c) Calculate $R, \kappa$ and $\rho_{0}$ from (4) to (7).

\section{ESTIMATION OF THE POSITION OF THE ACCELEROMETER}

In step (ii) from Section 2 the position of the accelerometer with respect to a robot fixed tool coordinate system is derived. From step (i) in Section 2 and the implementation in Section 3, the orientation and sensor parameters are known, hence the acceleration measured by the accelerometer has a known orientation. Next, the accelerometer's coordinate system $O x_{s} y_{s} z_{s}$ should be expressed in a coordinate system $O x_{b f} y_{b f} z_{b f}$ fixed to the robot.

Using a mathematical model of the robot motion it is possible to compute the acceleration, parameterised in some unknown parameters. To simplify the mathematical model for the acceleration and to make it possible to parameterise the unknown parameters, consider the case when the robot is in the configuration shown in Figure 3. The figure shows the vector $r_{s}$, the two coordinate systems $O x_{b f} y_{b f} z_{b f}$ and $O x_{s} y_{s} z_{s}$, the world fixed coordinate system $O x_{b} y_{b} z_{b}$ attached to the base of the robot, the coordinate system $O x_{w} y_{w} z_{w}$ fixed to the end of the robot arm, and the vector $a_{s} \triangleq \frac{d^{2}}{d t^{2}}\left(r_{s}\right)$ which describes the acceleration of $O x_{s} y_{s} z_{s}$. The mathematical expression for $a_{s}$ together with the measured acceleration are used in order to estimates the unknown parameters. The figure also shows a parameter $\theta$ describing the rotation between $O x_{b f} y_{b f} z_{b f}$ and $O x_{b} y_{b} z_{b}$, two known parameters $L_{1}$ and $L_{2}$ describing the arm lengths and three unknown parameters $l_{i}, i=1,2,3$ describing the vector $r_{s / w}$ in $O x_{w} y_{w} z_{w}$.

All the calculations are done in the world fixed coordinate system in order to obtain an expression for $\frac{d^{2}}{d t^{2}}\left(r_{s}\right)$. In a body fixed coordinate system $O x_{b f} y_{b f} z_{b f} \frac{d^{2}}{d t^{2}}\left(r_{s}\right)=0$. The notation $\left[r_{s}\right]_{i}$ is used to emphasise that $r_{s}$ is expressed in coordinate system $i$.

Figure (3) shows that $r_{s}$ can be written as a sum of two vectors,

$$
\left[r_{s}\right]_{b f}=\left[r_{w}\right]_{b f}+\left[r_{s / w}\right]_{b f}
$$

where

$$
\begin{aligned}
{\left[r_{s / w}\right]_{b f} } & =\left(\begin{array}{lll}
l_{3} & -l_{2} & -l_{1}
\end{array}\right)^{T} \\
{\left[r_{w}\right]_{b f} } & =\left(\begin{array}{lll}
L_{1} & 0 & L_{2}
\end{array}\right)^{T}
\end{aligned}
$$

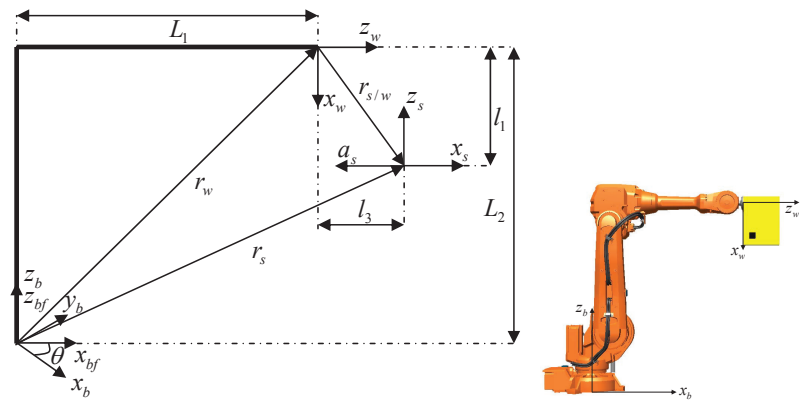

(a) From the side.

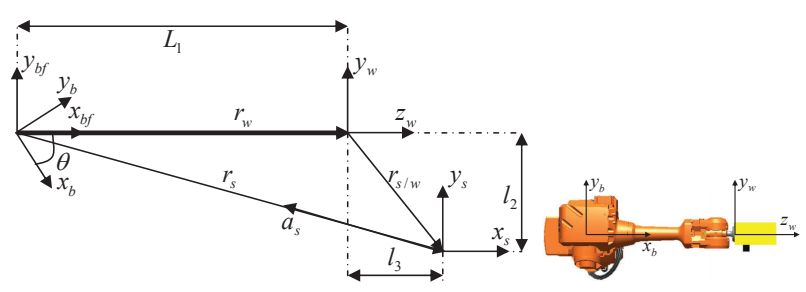

(b) From above.

Fig. 3. The first robot configuration for estimation of the mounting position. The black cube on the yellow box indicates the accelerometer, i.e., the origin of $O x_{s} y_{s} z_{s}$. The yellow box is attached to the robot in the point $\left(\begin{array}{lll}L_{1} & 0 & L_{2}\end{array}\right)^{T}$ expressed in $O x_{b f} y_{b f} z_{b f}$.

The transformation of $r_{s}$ from $O x_{b f} y_{b f} z_{b f}$ to $O x_{b} y_{b} z_{b}$ can be expressed as

$$
\left[r_{s}\right]_{b}=\left[Q_{b f / b}\right]_{b}\left(\left[r_{w}\right]_{b f}+\left[r_{s / w}\right]_{b f}\right),
$$

where

$$
\left[Q_{b f / b}\right]_{b}=\left(\begin{array}{ccc}
\cos \theta & -\sin \theta & 0 \\
\sin \theta & \cos \theta & 0 \\
0 & 0 & 1
\end{array}\right)
$$

is the rotation matrix that relates the coordinate system $O x_{b f} y_{b f} z_{b f}$ to $O x_{b} y_{b} z_{b} . \theta=\theta(t)$ is the angle relating $O x_{b} y_{b} z_{b}$ and $O x_{b f} y_{b f} z_{b f}$ according to Figure 3. Taking the derivative of $\left[r_{s}\right]_{b}$ with respect to time gives

$$
\frac{d}{d t}\left(\left[r_{s}\right]_{b}\right)=\frac{d}{d t}\left(\left[Q_{b f / b}\right]_{b}\right)\left(\left[r_{w}\right]_{b f}+\left[r_{s / w}\right]_{b f}\right) .
$$

The time derivative of the rotation matrix is given by (Spong et al., 2005)

$$
\frac{d}{d t}\left(\left[Q_{b f / b}\right]_{b}\right)=S(\omega)\left[Q_{b f / b}\right]_{b}
$$

where $\omega=\left(\begin{array}{lll}0 & 0 & \dot{\theta}\end{array}\right)^{T}$ and

$$
S(\omega)=\left(\begin{array}{ccc}
0 & -\dot{\theta} & 0 \\
\dot{\theta} & 0 & 0 \\
0 & 0 & 0
\end{array}\right)
$$

is a skew symmetric matrix. Hence, the time derivative of $\left[r_{s}\right]_{b}$ can be written

$$
\frac{d}{d t}\left(\left[r_{s}\right]_{b}\right)=S(\omega)\left[Q_{b f / b}\right]_{b}\left(\left[r_{w}\right]_{b f}+\left[r_{s / w}\right]_{b f}\right) .
$$

The second time derivative of $\left[r_{s}\right]_{b}$ becomes 


$$
\begin{aligned}
{\left[a_{s}\right]_{b}=} & \frac{d^{2}}{d t^{2}}\left(\left[r_{s}\right]_{b}\right)=\frac{d}{d t}(S(\omega))\left[Q_{b f / b}\right]_{b}\left(\left[r_{w}\right]_{b f}+\left[r_{s / w}\right]_{b f}\right) \\
& +S(\omega) \frac{d}{d t}\left(\left[Q_{b f / b}\right]_{b}\right)\left(\left[r_{w}\right]_{b f}+\left[r_{s / w}\right]_{b f}\right) \\
= & S(\dot{\omega})\left[Q_{b f} / b\right]_{b}\left(\left[r_{w}\right]_{b f}+\left[r_{s / w}\right]_{b f}\right) \\
& +S(\omega) S(\omega)\left[Q_{b f / b}\right]_{b}\left(\left[r_{w}\right]_{b f}+\left[r_{s / w}\right]_{b f}\right) \\
= & S(\omega) S(\omega)\left[Q_{b f / b}\right]_{b}\left(\left[r_{w}\right]_{b f}+\left[r_{s / w}\right]_{b f}\right),
\end{aligned}
$$

where $\dot{\omega}=\left(\begin{array}{lll}0 & 0 & 0\end{array}\right)^{T}$ follows from the assumption of constant angular velocity.

It now remains to transform the measured acceleration $a_{s}^{M}$ from $O x_{s} y_{s} z_{s}$ to $O x_{b} y_{b} z_{b}$. From Figure 3 it can be seen directly that

$$
\left[a_{s}^{M}\right]_{b f}=\left(\begin{array}{ccc}
a_{s, x}^{M} & a_{s, y}^{M} & 0
\end{array}\right)^{T},
$$

hence

$$
\left[a_{s}^{M}\right]_{b}=\left[Q_{b f / b}\right]_{b}\left[a_{s}^{M}\right]_{b f} .
$$

Equations (20) and (22) give

$$
\begin{gathered}
{\left[Q_{b f / b}\right]_{b}\left[a_{s}^{M}\right]_{b f}=S(\omega) S(\omega)\left[Q_{b f / b}\right]_{b}\left(\left[r_{w}\right]_{b f}+\left[r_{s / w}\right]_{b f}\right)} \\
\Leftrightarrow \\
{\left[a_{s}^{M}\right]_{b f}=\left[Q_{b f / b}\right]_{b}^{T} S(\omega) S(\omega)\left[Q_{b f / b}\right]_{b}\left(\left[r_{w}\right]_{b f}+\left[r_{s / w}\right]_{b f}\right)}
\end{gathered}
$$

since $\left[Q_{b f / b}\right]_{b}^{T}=\left[Q_{b f / b}\right]_{b}^{-1}$. Carrying out the matrix multiplication for the right-hand side of (23) gives

$$
\left[a_{s}^{M}\right]_{b f}=\left(\begin{array}{c}
-\dot{\theta}^{2}\left(L_{1}+l_{3}\right) \\
\dot{\theta}^{2} l_{2} \\
0
\end{array}\right),
$$

where (12), (13), (15) and (18) have been used. Equations (21) and (24) can now be written as a system of equations where $l_{2}$ and $l_{3}$ are unknown,

$$
\left(\begin{array}{cc}
0 & -\dot{\theta}^{2} \\
\dot{\theta}^{2} & 0
\end{array}\right)\left(\begin{array}{l}
l_{2} \\
l_{3}
\end{array}\right)=\left(\begin{array}{c}
a_{s, x}^{M}+\dot{\theta}^{2} L_{1} \\
a_{s, y}^{M}
\end{array}\right)
$$

It is thus possible to find $l_{2}$ and $l_{3}$ from (25) but unfortunately not $l_{1}$. Rotating the accelerometer according to Figure 4 will give information about $l_{1}$. The same calculations as before with

$$
\begin{aligned}
{\left[r_{s / w}\right]_{b f} } & =\left(\begin{array}{lll}
-l_{1} & -l_{2} & -l_{3}
\end{array}\right)^{T}, \\
{\left[r_{w}\right]_{b f} } & =\left(\begin{array}{lll}
L_{3} & 0 & L_{4}
\end{array}\right)^{T}, \\
{\left[a_{s}^{M}\right]_{b f} } & =\left(\begin{array}{lll}
a_{s, z}^{M} & a_{s, y}^{M} & 0
\end{array}\right)^{T},
\end{aligned}
$$

see Figure 4, give

$$
\left(\begin{array}{cc}
\dot{\theta}^{2} & 0 \\
0 & \dot{\theta}^{2}
\end{array}\right)\left(\begin{array}{l}
l_{1} \\
l_{2}
\end{array}\right)=\left(\begin{array}{c}
a_{s, z}^{M}+\dot{\theta}^{2} L_{3} \\
a_{s, y}^{M}
\end{array}\right) .
$$

Equations (25) and (29) can now be used to estimate the unknown parameters. Using (25) and (29) the estimation of $l_{2}$ uses approximately twice as much data than the estimation of $l_{1}$ and $l_{3}$. To get equal amount of data for each parameter, which gives a more accurate estimation, the robot configuration in Figure 5 is used, which gives

$$
\begin{aligned}
& {\left[r_{s / w}\right]_{b f}=\left(\begin{array}{lll}
l_{3} & -l_{1} & l_{2}
\end{array}\right)^{T},}
\end{aligned}
$$

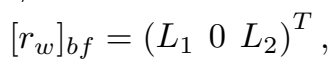

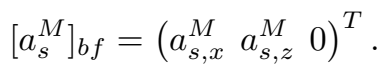

From (23) the following equation is obtained

$$
\left(\begin{array}{cc}
0 & -\dot{\theta}^{2} \\
\dot{\theta}^{2} & 0
\end{array}\right)\left(\begin{array}{l}
l_{1} \\
l_{3}
\end{array}\right)=\left(\begin{array}{c}
a_{s, x}^{M}+\dot{\theta}^{2} L_{1} \\
a_{s, z}^{M}
\end{array}\right)
$$
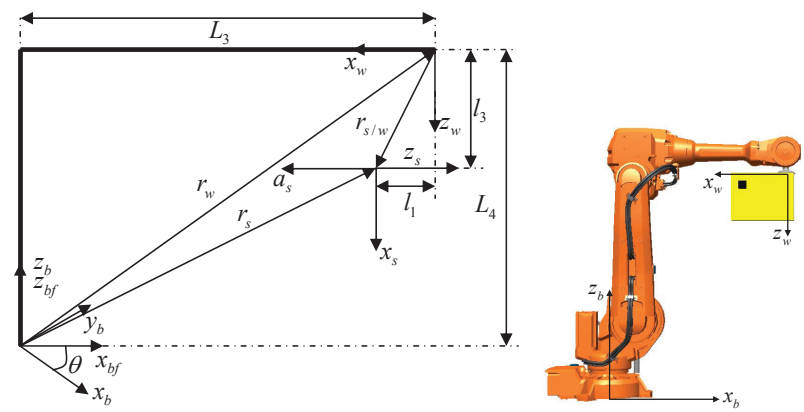

(a) From the side.

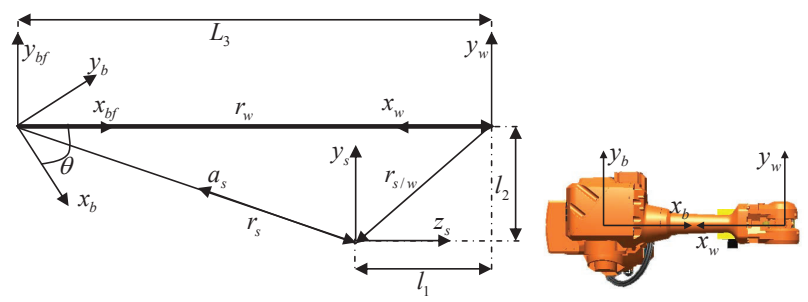

(b) From above.

Fig. 4. The second robot configuration for estimation of the mounting position. The black cube on the yellow box indicates the accelerometer, i.e., the origin of $O x_{s} y_{s} z_{s}$. The yellow box is attached to the robot in the point $\left(\begin{array}{lll}L_{3} & 0 & L_{4}\end{array}\right)^{T}$ expressed in $O x_{b f} y_{b f} z_{b f}$.
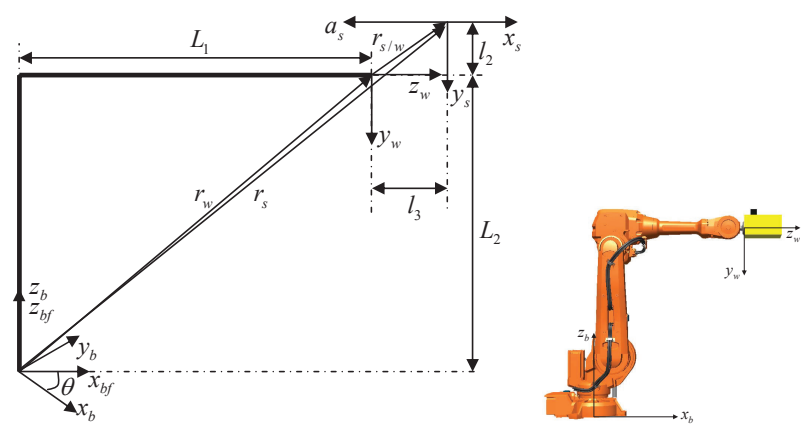

(a) From the side.
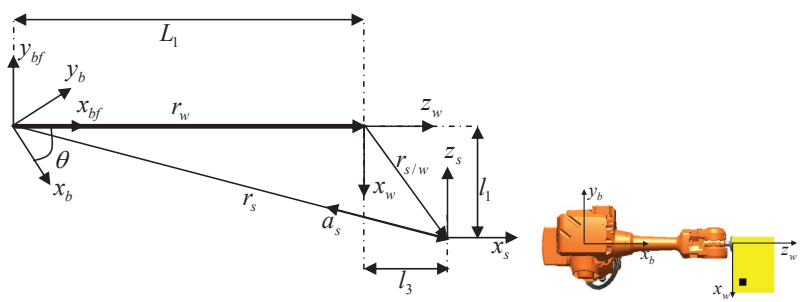

(b) From above.

Fig. 5. The third robot configuration for estimation of the mounting position. The black cube on the yellow box indicates the accelerometer, i.e., the origin of $O x_{s} y_{s} z_{s}$. The yellow box is attached to the robot in the point $\left(\begin{array}{lll}L_{1} & 0 & L_{2}\end{array}\right)^{T}$ expressed in $O x_{b f} y_{b f} z_{b f}$.

Equations (25), (29) and (33) can now be written as one system of equations according to 


$$
\underbrace{\left(\begin{array}{ccc}
0 & 0 & -\dot{\theta}_{c 1}^{2} \\
0 & \dot{\theta}_{c 1}^{2} & 0 \\
\dot{\theta}_{c 2}^{2} & 0 & 0 \\
0 & \dot{\theta}_{c 2}^{2} & 0 \\
0 & 0 & -\dot{\theta}_{c 3}^{2} \\
\dot{\theta}_{c 3}^{2} & 0 & 0
\end{array}\right)}_{A} \underbrace{\left(\begin{array}{l}
l_{1} \\
l_{2} \\
l_{3}
\end{array}\right)}_{l}=\underbrace{\left(\begin{array}{c}
a_{s, x, c 1}^{M}+\dot{\theta}_{c 1}^{2} L_{1} \\
a_{s, y, c 1}^{M} \\
a_{s, z, c 2}^{M}+\dot{\theta}_{c 2}^{2} L_{3} \\
a_{s, y, c 2}^{M} \\
a_{s, x, c 3}^{M}+\dot{\theta}_{c 3}^{2} L_{1} \\
a_{s, z, c 3}^{M}
\end{array}\right)}_{b},
$$

where index $c i, i=1,2,3$ indicates from which robot configuration the measurements come from. Equation (34) has more rows than unknowns, hence the solution to (34) is given by the solution to the optimisation problem

$$
\underset{l}{\arg \min }\|b-A l\|_{2}^{2}
$$

which has the analytical solution

$$
\hat{l}=\left(A^{T} A\right)^{-1} A^{T} b .
$$

There exist better numerical solutions to (34) than (36), e.g. $l=A \backslash b$ in MATLAB. The procedure to estimate the position of the accelerometer is summarised in Algorithm 2. Algorithm 2. Estimation of the mounting position

a) Measure the acceleration of the tool $\left[a_{s}^{M}\right]_{s}$ and the angular velocity $\dot{\theta}$ for the three different configurations in Figures 3,4 and 5 when $\theta$ varies from $\theta_{\text {min }}$ to $\theta_{\max }$ with constant angular velocity.

b) Construct $A$ and $b$ in (34).

c) Solve (34) with respect to $l$, for example according to $(36)$.

\section{EXPERIMENTAL RESULTS}

In this section the proposed orientation and position estimation method described in the two algorithms in Sections 3 and 4 is evaluated using experimental data. For Algorithm 1, the data, i.e., the acceleration values, are collected during $4 \mathrm{~s}$ for each one of the six configurations in Figure 2 using a sample rate of $2 \mathrm{kHz}$. For Algorithm 2, the arm angular velocity $\dot{\theta}$ for joint 1 and the acceleration measurements are collected when the robot is in the three different configurations according to Figures 3, 4 and 5. The arm angular velocity for joint 1 is computed from the motor angular velocity $\dot{\theta}_{m}$ using,

$$
\dot{\theta}_{m}=\tau \dot{\theta},
$$

where $\tau$ is the gear ratio. In the position estimation experiments data are collected during $4 \mathrm{~s}$ in each one of the three configurations, but it is only the constant angular velocity part of the data that is used. The same sample rate as before is used, i.e., $2 \mathrm{kHz}$. The accelerometer used in the experiments is a triaxial accelerometer from Crossbow Technology, with a range of $\pm 2 \mathrm{~g}$, and a sensitivity of approximately $1 \mathrm{~V} / \mathrm{g}$ Crossbow Technology (2004). The accelerometer is connected to the measurement system of the robot controller, and hence the acceleration and motor angular velocity can be synchronised and measured with the same sampling rate.

Three different mounting positions and different orientations of the accelerometer have been used for evaluation of Algorithms 1 and 2. The actual physical orientation of the accelerometer was measured using a protractor, see Figure 6 , where the orientation of the desired coordinate system for the accelerometer also is shown.
1

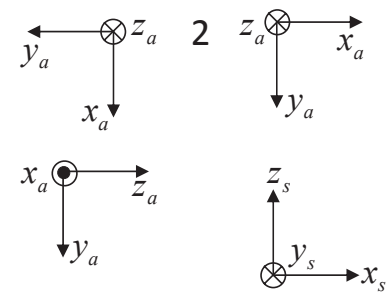

Fig. 6. Orientation for the three mounting positions that were used to evaluate the two algorithms. The orientation of the desired coordinate system is also shown.

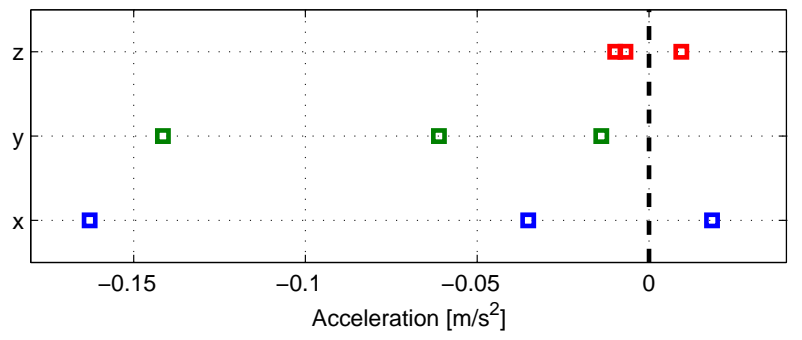

Fig. 7. Diagram of the transformation errors in the $\mathrm{x}-\mathrm{y}, \mathrm{y}$ and z-direction for (1) in configuration 1 (Figure 2) for all three test cases.

Algorithm 1 was applied to the three test cases presented above and the result $\hat{R}, \hat{\kappa}$ and $\hat{\rho}_{0}$ can be seen in Table 1 . From Figure 6 it can be seen that the rotation matrix $R$ in (1) should resemble

$$
R^{1}=\left(\begin{array}{ccc}
0 & -1 & 0 \\
0 & 0 & 1 \\
-1 & 0 & 0
\end{array}\right), R^{2}=\left(\begin{array}{ccc}
1 & 0 & 0 \\
0 & 0 & 1 \\
0 & -1 & 0
\end{array}\right), R^{3}=\left(\begin{array}{ccc}
0 & 0 & 1 \\
-1 & 0 & 0 \\
0 & -1 & 0
\end{array}\right) \text {. }
$$

The superscript indicates the test number. A rotational difference between the measured rotation matrix $R^{i}$ and the estimated matrix $\hat{R}^{i}$ can be computed using the corresponding unit quaternions $\mathfrak{q}^{i}$ and $\hat{\mathfrak{q}}^{i}$. The rotation angle $\vartheta^{i}$ of $\mathfrak{q}_{\Delta}^{i}$ from $\mathfrak{q}_{\Delta}^{i}=\left(\mathfrak{q}^{i}\right)^{-1} * \hat{\mathfrak{q}}^{i}$, which should be small ${ }^{2}$, is a good measure of the difference between $R^{i}$ and $\hat{R}^{i}$. See e.g. Sciavicco and Siciliano (2000) for a short introduction to quaternions. The resulting rotation angle $\vartheta^{i}$ for the three test cases can be seen i Table 2. The difference is small in all cases.

It is more difficult to obtain true values for the parameters $\kappa$ and $\rho_{0}$. To verify them, the measured acceleration for all three test cases in configuration 1, in Figure 2, is transformed from $O x_{a} y_{a} z_{a}$ to $O x_{s} y_{s} z_{s}$, which results in three constant signals $a_{s, x}^{M}, a_{s, y}^{M}$ and $a_{s, z}^{M}$ for the three axes of the accelerometer. Figure 2 shows that the measured acceleration in frame $O x_{s} y_{s} z_{s}$ should resemble $a_{s, x}=0$, $a_{s, y}=0$ and $a_{s, z}=g$. Subtracting $a_{s, j}$ from the mean of $a_{s, j}^{M}, j=x, y, z$, gives an error for the transformed acceleration. A diagram of the errors for each coordinate axis in $O x_{s} y_{s} z_{s}$ is shown in Figure 7. The errors are small and, as expected, the errors are larger in $x$ and $y$ due to the higher sensitivity to orientation errors in these axis when measuring gravity along the $z$-axis. The bias in $x$ can also be explained by a systematic error in orientation due to the robot elasticity and gravitational force acting on the robot in the evaluation position, see Figure 1.

\footnotetext{
2 The matrices are identical if $\vartheta=0^{\circ}$.
} 
Table 1. Estimated parameters in (1) using

Algorithm 1 for three different test cases.

\begin{tabular}{|c|c|c|c|c|c|}
\hline Test & $\widehat{\hat{\kappa}}$ & $\hat{\rho}_{0}$ & & $\hat{R}$ & \\
\hline 1 & 9.91 & $\left(\begin{array}{c}25.05 \\
-23.75 \\
24.26\end{array}\right)$ & $\left(\begin{array}{l}-0.0138 \\
-0.0094 \\
-0.9998\end{array}\right.$ & $\begin{array}{c}-0.9998 \\
-0.0168 \\
0.0140\end{array}$ & $\left.\begin{array}{c}-0.0170 \\
0.9998 \\
-0.0092\end{array}\right)$ \\
\hline 2 & 9.91 & $\left(\begin{array}{c}-23.89 \\
-24.03 \\
25.11\end{array}\right)$ & $\left(\begin{array}{r}0.9999 \\
0.0129 \\
-0.0073\end{array}\right.$ & $\begin{array}{l}-0.0070 \\
-0.0276 \\
-0.9996\end{array}$ & $\left.\begin{array}{c}-0.0131 \\
0.9995 \\
-0.0275\end{array}\right)$ \\
\hline 3 & 9.91 & $\left(\begin{array}{c}-24.46 \\
24.86 \\
23.74\end{array}\right)$ & $\left(\begin{array}{c}0.0169 \\
-0.9992 \\
0.0353\end{array}\right.$ & $\begin{array}{l}-0.0135 \\
-0.0355 \\
-0.999\end{array}$ & $\left.\begin{array}{c}0.9998 \\
0.0164 \\
-0.0145\end{array}\right)$ \\
\hline
\end{tabular}

Table 2. The rotation angle $\vartheta$ indicates how close the estimated and measured rotation matrices are to each other.

\begin{tabular}{c||ccc} 
Test & 1 & 2 & 3 \\
\hline$\vartheta$ & $1.4^{\circ}$ & $1.8^{\circ}$ & $2.4^{\circ}$
\end{tabular}

Table 3. Estimated positions $\hat{l}$ of the accelerometer, the error $\Delta$ relative the measured position $l^{M}$, and the standard deviation for $\hat{l}$.

\begin{tabular}{ccccc}
\hline Test & Est. pos. $(\hat{l})[\mathrm{cm}]$ & $\Delta=\hat{l}-l^{M}[\mathrm{~cm}]$ & Std. for $\hat{l}[\mathrm{~cm}]$ \\
\hline \hline 1 & $\left(\begin{array}{lllll}35.2 & 6.3 & 15.5\end{array}\right)^{T}$ & $\left(\begin{array}{llll}0.2 & 2.3 & -1.0\end{array}\right)^{T}$ & $\left(\begin{array}{llll}0.4 & 0.5 & 0.5\end{array}\right)^{T}$ \\
2 & $\left(\begin{array}{llll}14.2 & 5.8 & 16.9\end{array}\right)^{T}$ & $\left(\begin{array}{llll}-0.3 & -1.2 & 1.8\end{array}\right)^{T}$ & $\left(\begin{array}{llll}0.3 & 0.3 & 0.3\end{array}\right)^{T}$ \\
3 & $\left(\begin{array}{lllll}29.2 & 1.6 & 5.9\end{array}\right)^{T}$ & $\left(\begin{array}{llll}2.2 & 1.6 & 0.4\end{array}\right)^{T}$ & $\left(\begin{array}{llll}0.4 & 0.4 & 0.4\end{array}\right)^{T}$ \\
\hline
\end{tabular}

The estimated position $\hat{l}$ and the error $\Delta$ between $\hat{l}$ and the measured position $l^{M}$, for the three test cases can be seen in Table 3. The position was always measured using a tape measure to the centre of the accelerometer, since the position of the origin of the accelerometer's coordinate system inside the sensor is unspecified.

If the measured $\dot{\theta}$ is assumed to be with without noise, which is a reasonable assumption for the robot system, then $\hat{l}$ is linear dependent of the noise, originating from the measured acceleration, according to (36). The covariance matrix for $\hat{l}$ can therefore be calculated as

$$
\operatorname{Cov}(\hat{l})=\left(A^{T} A\right)^{-T} \operatorname{Cov}(b) .
$$

$\operatorname{Cov}(b)$ is a scalar and the structure of $A$ implies that $\left(A^{T} A\right)^{-T}$ is a diagonal matrix, hence $\operatorname{Cov}(\hat{l})$ is diagonal. The square root of the diagonal elements in $\operatorname{Cov}(\hat{l})$, i.e., the standard deviation, of the estimated position are presented in Table 3.

Considering the accuracy of the measurements and the uncertainty of the origin of the accelerometer coordinate system the result in Table 3 is considered as acceptable. The actual requirement of the result, in terms of position and orientation accuracy, will depend on the application where the accelerometer is used. A more detailed investigation of the requirement for the accuracy in the dynamic position and orientation estimation of the tool position, such as described in Axelsson et al. (2012); Axelsson (2012), is left as future work.

\section{CONCLUSIONS}

A method to find the position and orientation of a triaxial accelerometer mounted on a six DOF robot is presented. The method is divided into two main steps, where in the first step, the orientation is estimated by finding the transformation from the actual coordinate system of the accelerometer, with unknown orientation, to a new coordinate system with known orientation. It is also possible to find the sensitivity and the bias parameters. The estimation of the orientation is based on static measurements of the gravity vector when the accelerometer is placed in different orientations using the six DOF robot arm. In the second step of the method, the mounting position of the accelerometer in a robot fixed coordinate system is computed using several experiments where the robot is moving with constant speed. Finally, the method is evaluated on experimental data. The resulting position and orientation accuracy are evaluated using measurements on the physical system. The orientation error is in the range 1 to 2 degrees and the position error is in average $\bar{\Delta}=\left(\begin{array}{lll}0.7 & 0.9 & 0.4\end{array}\right)^{T} \mathrm{~cm}$, where $\Delta$ is the error relative the measured position $l^{M}$. The accuracy is sufficient in experiments with dynamic position and orientation estimation of the tool position using sensor fusion methods, such as extended Kalman filter and particle filter.

\section{REFERENCES}

Axelsson, P. (2012). Evaluation of six different sensor fusion methods for an industrial robot using experimental data. In Proceedings the 10th International IFAC Symposium on Robot Control. Dubrovnik, Croatia.

Axelsson, P., Karlsson, R., and Norrlöf, M. (2012). Tool position estimation of a flexible industrial robot using recursive Bayesian methods. In Proceedings of the IEEE Conference on Robotics and Automation 2012. St. Paul, Minnesota, USA.

Crossbow Technology (2004). Accelerometers, High Sensitivity, LF Series, CXL02LF3. Http://www.xbow.com.

Horn, B.K.P. (1987). Closed-form solution of absolute orientation using unit quaternions. Journal of the Optical Society of America, $4(4), 629-642$.

Horn, B.K.P., Hilden, H.M., and Negahdaripour, S. (1988). Closedform solution of absolute orientation using orthonormal matrices. Journal of the Optical Society of America, 5(7), 1127-1135.

Renk, E.L., Collins, W., Rizzo, M., Lee, F., and Bernstein, D.S. (2005). Calibrating a triaxial accelerometer-magnetometer-using robotic actuation for sensor reorientation during data collection. Control Systems Magazine, 25(6), 86-95.

Sciavicco, L. and Siciliano, B. (2000). Modelling and Control of Robot Manipulators. Springer, London, UK, second edition.

Spong, M.W., Hutchinson, S., and Vidyasagar, M. (2005). Robot Modeling and Control. John Wiley \& Sons.

Won, S.h.P. and Golnaraghi, F. (2010). A triaxial accelerometer calibration method using a mathematical model. IEEE Transactions on Instrumentation and Measurement, 59(8), 2144-2153. 